Bull. Korean Math. Soc. 49 (2012), No. 5, pp. 989-996

http://dx.doi.org/10.4134/BKMS.2012.49.5.989

\title{
AMALGAMATED DUPLICATION OF SOME SPECIAL RINGS
}

\author{
Elham Tavasoli, Maryam Salimi, and Abolfazl Tehranian
}

Abstract. Let $R$ be a commutative Noetherian ring and let $I$ be an ideal of $R$. In this paper we study the amalgamated duplication ring $R \bowtie I$ which is introduced by D'Anna and Fontana. It is shown that if $R$ is generically Cohen-Macaulay (resp. generically Gorenstein) and $I$ is generically maximal Cohen-Macaulay (resp. generically canonical module), then $R \bowtie I$ is generically Cohen-Macaulay (resp. generically Gorenstein). We also defined generically quasi-Gorenstein ring and we investigate when $R \bowtie I$ is generically quasi-Gorenstein. In addition, it is shown that $R \bowtie I$ is approximately Cohen-Macaulay if and only if $R$ is approximately Cohen-Macaulay, provided some special conditions. Finally it is shown that if $R$ is approximately Gorenstein, then $R \bowtie I$ is approximately Gorenstein.

\section{Introduction}

Throughout this paper all rings are considered commutative with identity element and all ring homomorphisms are unital. In [8], D'Anna and Fontana considered a different type of construction obtained involving a ring $R$ and an ideal $I \subset R$ that is denoted by $R \bowtie I$, called amalgamated duplication, and it is defined as the following subring of $R \times R$ :

$$
R \bowtie I=\{(r, r+i) \mid r \in R, i \in I\} .
$$

In [6] D'Anna showed that if $R$ is a Noetherian local ring, then $R \bowtie I$ is Cohen-Macaulay if and only if $R$ is Cohen-Macaulay and $I$ is maximal CohenMacaulay. In [1] it is shown that if $R$ is a Noetherian local ring, then $R \bowtie I$ is Gorenstein if and only if $R$ is Cohen-Macaulay and $I$ is a canonical module for $R$, and then $R / I$ is Cohen-Macaulay of dimension $\operatorname{dim}(R)-1$. In this paper it is shown that if $R \bowtie I$ is a Gorenstein ring where $I$ is a non-zero flat ideal of Noetherian zero dimensional ring $R$, then $R$ is Gorenstein (see Proposition 2.2). Recently, the authors in [4] showed that if $R$ is a Noetherian local ring and $I$ is a proper ideal of $R$ such that $\operatorname{Ann}_{R}(I)=0$, then $R \bowtie I$ is a quasi-Gorenstein

Received May 18, 2011.

2010 Mathematics Subject Classification. $13 \mathrm{H} 10$.

Key words and phrases. amalgamated duplication, generically Cohen-Macaulay, generically Gorenstein, approximately Cohen-Macaulay, approximately Gorenstein. 
ring if and only if $\widehat{R}$ satisfies Serre's condition $\left(S_{2}\right)$ and $I$ is a canonical ideal of $R$.

Recall that a Noetherian ring $R$ is called generically Cohen-Macaulay (resp. generically Gorenstein) if the ring $R_{\mathfrak{p}}$ is Cohen-Macaulay (resp. Gorenstein) for all $\mathfrak{p} \in \operatorname{Ass}(R)$. Every Cohen-Macaulay (resp. Gorenstein) ring is also generically Cohen-Macaulay (resp. generically Gorenstein) and every Artinian generically Cohen-Macaulay (resp. generically Gorenstein) ring is Cohen-Macaulay (resp. Gorenstein). In Section 2 we define a generically quasi-Gorenstein ring and we investigate when $R \bowtie I$ is a generically Cohen-Macaulay (resp. generically Gorenstein, generically quasi-Gorenstein) ring (see Theorem 2.8 and Proposition 2.9).

In [9] Goto defined approximately Cohen-Macaulay ring and in [13] the authors examined how this property transfers under flat maps and tensor product operations. In [10] Hochster defined approximately Gorenstein ring. In Section 3 we provide necessary and sufficient conditions which led $R \bowtie I$ be an approximately Cohen-Macaulay (resp. approximately Gorenstein) ring (see Proposition 3.2 and Theorem 3.4).

\section{Generically Cohen-Macaulay, generically Gorenstein and generically quasi-Gorenstein rings}

As general reference for terminology and well-known results, we refer the reader to [5]. This section deals with some general results about generically Cohen-Macaulay, generically Gorenstein and generically quasi-Gorenstein properties of a general construction, introduced in [8], called amalgamated duplication of a ring along an ideal.

Let $R$ be a commutative ring with unit element 1 and let $I$ be a proper ideal of $R$. Set

$$
R \bowtie I=\{(r, s) \mid r, s \in R, s-r \in I\} .
$$

It is easy to check that $R \bowtie I$ is a subring, with unit element $(1,1)$, of $R \times R$ (with the usual componentwise operations) and that $R \bowtie I=\{(r, r+i) \mid r \in$ $R, i \in I\}$. In the following we bring some main properties of the $\operatorname{ring} R \bowtie I$ from $[6]$.

Proposition 2.1. Let $R$ be a ring and let $I$ be an ideal of $R$. Then the following statements hold.

(1) The map $f: R \oplus I \rightarrow R \bowtie I$ defined by $f((r, i))=(r, r+i)$ is an $R$ isomorphism. Moreover, there is a split exact sequence of $R$-modules

$$
0 \rightarrow R \stackrel{\varphi}{\rightarrow} R \bowtie I \stackrel{\psi}{\rightarrow} I \rightarrow 0,
$$

where $\varphi(r)=(r, r)$ for all $r \in R$, and $\psi((r, s))=s-r$ for all $(r, s) \in$ $R \bowtie I$. We also have the short exact sequence of $R$-modules:

$$
0 \rightarrow I \stackrel{\psi^{\prime}}{\rightarrow} R \bowtie I \stackrel{\varphi^{\prime}}{\rightarrow} R \rightarrow 0,
$$


where $\psi^{\prime}(i)=(0, i)$ and $\varphi^{\prime}((r, s))=r$ for every $r \in R$ and $(r, s) \in R \bowtie$ I. Note that the exact sequence (b) is also a sequence of $R \bowtie I$-module, while the other one is not.

(2) Let $\mathfrak{p}$ be a prime ideal of $R$ and set:

$$
\begin{aligned}
& \mathfrak{p}_{0}=\{(p, p+i) \mid p \in \mathfrak{p}, i \in I \cap \mathfrak{p}\}, \\
& \mathfrak{p}_{1}=\{(p, p+i) \mid p \in \mathfrak{p}, i \in I\}, \text { and } \\
& \mathfrak{p}_{2}=\{(p+i, p) \mid p \in \mathfrak{p}, i \in I\} .
\end{aligned}
$$

(a) If $I \subseteq \mathfrak{p}$, then $\mathfrak{p}_{0}=\mathfrak{p}_{1}=\mathfrak{p}_{2}$ is a prime ideal of $R \bowtie I$ and it is the unique prime ideal of $R \bowtie I$ lying over $\mathfrak{p}$ and $(R \bowtie I)_{\mathfrak{p}_{0}} \cong R_{\mathfrak{p}} \bowtie$ $I_{\mathfrak{p}}$.

(b) If $I \nsubseteq \mathfrak{p}$, then $\mathfrak{p}_{1} \neq \mathfrak{p}_{2}$ and $\mathfrak{p}_{1} \cap \mathfrak{p}_{2}=\mathfrak{p}_{0}$. Moreover, $\mathfrak{p}_{1}$ and $\mathfrak{p}_{2}$ are the only prime ideals of $R \bowtie I$ lying over $\mathfrak{p}$, and $(R \bowtie I)_{\mathfrak{p}_{1}} \cong$ $R_{\mathfrak{p}} \cong(R \bowtie I)_{\mathfrak{p}_{2}}$.

(3) $R$ and $R \bowtie I$ have the same Krull dimension and if $R$ is a local ring with maximal ideal $\mathfrak{m}$, then $R \bowtie I$ is local with maximal ideal $\mathfrak{m}_{0}=$ $\{(r, r+i) \mid r \in \mathfrak{m}, i \in I\}$. Also, if $R$ is a Noetherian ring, then $R \bowtie I$ is a finitely generated $R$-module.

In [6, Discussion 10], D'Anna showed that if $R$ is a local ring of dimension $d$ and $I$ is a non-unit ideal of $R$, then the $\operatorname{ring} R \bowtie I$ is Cohen-Macaulay if and only if $R$ is Cohen-Macaulay and $I$ is a maximal Cohen-Macaulay $R$ module. Recently in [1, Theorem 1.8], it is shown that if $R$ is a Noetherian local ring, then $R \bowtie I$ is Gorenstein if and only if $R$ is Cohen-Macaulay and $I$ is a canonical module for $R$, and then $R / I$ is Cohen-Macaulay of dimension $\operatorname{dim}(R)-1$. In the following proposition we suppose that $R \bowtie I$ is Gorenstein and we would like to know when $R$ is Gorenstein.

Proposition 2.2. Let I be a non-zero flat ideal of Noetherian zero dimensional ring $R$. If $R \bowtie I$ is a Gorenstein ring, then $R$ is Gorenstein.

Proof. By Proposition 2.1(3), $\operatorname{dim}(R \bowtie I)=\operatorname{dim}(R)=0$ and so $R \bowtie I$ is self-injective. Hence by [14, Corollary 3.4], $\mathrm{id}_{R}(R \bowtie I)=\mathrm{fd}_{R}(R \bowtie I)$. Now by assumption $I$ is a flat ideal of $R$, so $R \bowtie I$ is a flat $R$-module. Therefore $R \bowtie I$ is an injective $R$-module and hence for every $R$-module $M$ and every integer $i \geq 1$, we have

$$
\begin{aligned}
0 & =\operatorname{Ext}_{R}^{i}(M, R \bowtie I) \\
& \cong \operatorname{Ext}_{R}^{i}(M, R) \oplus \operatorname{Ext}_{R}^{i}(M, I) .
\end{aligned}
$$

So for every $R$-module $M$ and for all $i \geq 1$, we have $\operatorname{Ext}_{R}^{i}(M, R)=0$. Hence $R$ is self-injective and therefore $R$ is Gorenstein, $\operatorname{since} \operatorname{dim}(R)=0$.

We recall the notion of quasi-Gorenstein ring due to Platte and Storch in $[12]$. 
Definition 2.3. A local $\operatorname{ring} R$ is said to be a quasi-Gorenstein ring if a canonical module of $R$ exists and is a free $R$-module (of rank one). This is equivalent to saying that $H_{\mathfrak{m}}^{d}(R) \cong E_{R}(R / \mathfrak{m})$, where $d=\operatorname{dim} R$ and $\mathfrak{m}$ is the maximal ideal of $R$.

The ring $R$ is Gorenstein if and only if it is quasi-Gorenstein and CohenMacaulay. In [4, Theorem 3.3], it is shown that if $R$ is a Noetherian local ring and $I$ is a proper ideal of $R$ such that $\operatorname{Ann}_{R}(I)=0$, then $R \bowtie I$ is a quasi-Gorenstein ring if and only if $\widehat{R}$ satisfies Serre's condition $\left(S_{2}\right)$ and $I$ is a canonical ideal of $R$.

Recall that a Noetherian ring $R$ is called generically Cohen-Macaulay (resp. generically Gorenstein) if the ring $R_{\mathfrak{p}}$ is Cohen-Macaulay (resp. Gorenstein) for all $\mathfrak{p} \in \operatorname{Ass}(R)$. Every Cohen-Macaulay (resp. Gorenstein) ring is also generically Cohen-Macaulay (resp. generically Gorenstein) and every Artinian generically Cohen-Macaulay (resp. generically Gorenstein) ring is Cohen-Macaulay (resp. Gorenstein). We are ready now to introduce generically quasi-Gorenstein ring.

Definition 2.4. Let $R$ be a Noetherian local ring. Then $R$ is called generically quasi-Gorenstein if the ring $R_{\mathfrak{p}}$ is quasi-Gorenstein for all $\mathfrak{p} \in \operatorname{Ass}(R)$.

According to [2, Corollary 2.4], the localization of every quasi-Gorenstein ring is quasi-Gorenstein. Therefore every quasi-Gorenstein ring is generically quasi-Gorenstein. It is straightforward to see that if $R$ is a zero dimensional local ring, then $R$ is quasi-Gorenstein if and only if $R$ is generically quasiGorenstein. It is routine to show that a Noetherian local ring $R$ is generically Gorenstein if and only if $R$ is generically quasi-Gorenstein and generically Cohen-Macaulay.

We are interested in understanding when $R \bowtie I$ is generically CohenMacaulay (resp. generically Gorenstein, generically quasi-Gorenstein). In the following lemma we investigate the associated prime ideals of the ring $R \bowtie I$.

Lemma 2.5. Let $R$ be a Noetherian ring and let $I$ be a proper ideal of $R$. Consider the ring homomorphism $\varphi: R \rightarrow R \bowtie I$, where $\varphi(r)=(r, r)$. Then the following statements hold.

(i) If $\mathfrak{p} \in A s s(R \bowtie I)$, then $\varphi^{-1}(\mathfrak{p}) \in \operatorname{Ass}(R)$.

(ii) If $\mathfrak{q} \in A s s(R)$, then there exists $\mathfrak{p} \in A$ ss $(R \bowtie I)$ such that $\varphi^{-1}(\mathfrak{p})=\mathfrak{q}$.

Proof. (i) The exact sequence $0 \rightarrow I \rightarrow R \bowtie I \rightarrow R \rightarrow 0$ of $R \bowtie I$-modules implies that

$$
\begin{aligned}
\operatorname{Ass}(R \bowtie I) & \subseteq \operatorname{Ass}_{R \bowtie I}(I) \cup \operatorname{Ass}_{R \bowtie I}(R) \\
& =\operatorname{Ass}_{R \bowtie I}(R) .
\end{aligned}
$$

So by assumption $\mathfrak{p} \in \operatorname{Ass}_{R \bowtie I}(R)$. By [11, Exercise 6.7] we have $\varphi^{-1}(\mathfrak{p}) \in$ Ass $(R)$, since $R$ is a finitely generated $R \bowtie I$-module. 
(ii) From the $R$-monomorphism $\varphi: R \rightarrow R \bowtie I$, we have $\operatorname{Ass}_{R}(R) \subseteq$ $\operatorname{Ass}_{R}(R \bowtie I)$. So by assumption $\mathfrak{q} \in \operatorname{Ass}_{R}(R \bowtie I)$ and by [11, Exercise 6.7] there exists $\mathfrak{p} \in \operatorname{Ass}_{R \bowtie I}(R \bowtie I)$ such that $\varphi^{-1}(\mathfrak{p})=\mathfrak{q}$.

Definition 2.6. A finitely generated $R$-module $M$ is called generically maximal Cohen-Macaulay (resp. generically canonical module) if the $R_{\mathfrak{p}}$-module $M_{\mathfrak{p}}$ is maximal Cohen-Macaulay (resp. canonical module) for all $\mathfrak{p} \in \operatorname{Ass}(R)$.

Definition 2.7. The ring $R$ is called generically $\left(S_{n}\right)$ if $R_{\mathfrak{p}}$ satisfies Serre's condition $\left(S_{n}\right)$ for all $\mathfrak{p} \in \operatorname{Ass}(R)$.

Theorem 2.8. Let $R$ be a Noetherian ring and let $I$ be a proper ideal of $R$. Then the following statements hold.

(i) If $R \bowtie I$ is generically Cohen-Macaulay, then $R$ is generically CohenMacaulay.

(ii) If $R$ is generically Cohen-Macaulay (resp. generically Gorenstein) and $I$ is generically maximal Cohen-Macaulay (resp. generically canonical module), then $R \bowtie I$ is generically Cohen-Macaulay (resp. generically Gorenstein).

(iii) If $R$ is generically quasi-Gorenstein and $I$ is a generically canonical ideal of $R$, then $R \bowtie I$ is generically quasi-Gorenstein.

(iv) If $\operatorname{Ann}_{R}(I)=0$, then $R$ is generically $\left(S_{2}\right)$ provided that $R \bowtie I$ is generically quasi-Gorenstein.

Proof. We prove items (iii) and (iv). The proof of the others is similar.

(iii) Let $\mathfrak{p} \in \operatorname{Ass}(R \bowtie I)$. By Lemma 2.5, $\mathfrak{q}=\varphi^{-1}(\mathfrak{p}) \in \operatorname{Ass}(R)$. According to Proposition 2.1(2), we have the following two cases:

Case (1). If $I \subseteq \mathfrak{q}$, then $(R \bowtie I)_{\mathfrak{p}} \cong R_{\mathfrak{q}} \bowtie I_{\mathfrak{q}}$. By assumption $I_{\mathfrak{q}}$ is a canonical ideal and $R_{\mathfrak{q}}$ is quasi-Gorenstein. Therefore $R_{\mathfrak{q}}$ satisfies Serre's condition $\left(S_{2}\right)$ by [3, Remark 1.4]. Hence $\widehat{R_{\mathfrak{q}}}$ satisfies Serre's condition $\left(S_{2}\right)$ by [3, Proposition 1.2]. Now according to [4, Theorem 3.3], $(R \bowtie I)_{\mathfrak{p}}$ is quasi-Gorenstein. Case (2). If $I \nsubseteq \mathfrak{q}$, then $(R \bowtie I)_{\mathfrak{p}} \cong R_{\mathfrak{q}}$. So $(R \bowtie I)_{\mathfrak{p}}$ is quasi-Gorenstein.

(iv) Let $\mathfrak{q} \in \operatorname{Ass}(R)$. By Lemma 2.5, there exists $\mathfrak{p} \in \operatorname{Ass}(R \bowtie I)$ such that $\varphi^{-1}(\mathfrak{p})=\mathfrak{q}$ and, by Proposition $2.1(2)$, we have the following two cases: Case (1). If $I \subseteq \mathfrak{q}$, then $(R \bowtie I)_{\mathfrak{p}} \cong R_{\mathfrak{q}} \bowtie I_{\mathfrak{q}}$. So by assumption $R_{\mathfrak{q}} \bowtie I_{\mathfrak{q}}$ is quasi-Gorenstein. Therefore by [4, Theorem 3.3], $\widehat{R_{\mathfrak{q}}}$ satisfies Serre's condition $\left(S_{2}\right)$ and so $R_{\mathfrak{q}}$ satisfies Serre's condition $\left(S_{2}\right)$ by [3, Proposition 1.2].

Case $(2)$. If $I \nsubseteq \mathfrak{q}$, then $(R \bowtie I)_{\mathfrak{p}} \cong R_{\mathfrak{q}}$. So $R_{\mathfrak{q}}$ satisfies Serre's condition $\left(S_{2}\right)$, by [3, Remark 1.4].

Proposition 2.9. Let $R$ be a Cohen-Macaulay ring and let $I$ be a non-zero ideal of $R$ such that $I_{\mathfrak{q}}$ is a flat $R_{\mathfrak{q}}$-module for all $\mathfrak{q} \in A s s(R)$. If $R \bowtie I$ is generically Gorenstein, then $R$ is generically Gorenstein.

Proof. Note that $\operatorname{dim}\left(R_{\mathfrak{q}}\right)=0$ for all $\mathfrak{q} \in \operatorname{Ass}(R)$, since $R$ is Cohen-Macaulay. The assertion follows by Propositions 2.2 and $2.1(3)$. 


\section{Approximately Cohen-Macaulay and approximately Gorenstein rings}

In this section we study when $R \bowtie I$ is approximately Cohen-Macaulay and when it is approximately Gorenstein. To state the first result of this section, we need the notion of approximately Cohen-Macaulay ring due to Goto in [9].

Definition 3.1. The local ring $(R, \mathfrak{m})$ is called an approximately CohenMacaulay ring if either $\operatorname{dim}(R)=0$ or there exists an element $a$ of $\mathfrak{m}$ such that $R / a^{n} R$ is a Cohen-Macaulay ring of dimension $\operatorname{dim}(R)-1$ for every integer $n>0$.

It is straightforward to see that a Cohen-Macaulay local ring $R$ is approximately Cohen-Macaulay and the converse is true when $\operatorname{dim}(R)=0$. Also Goto in [9, Corollary 2.8], showed that if $(R, \mathfrak{m})$ is an approximately CohenMacaulay local ring such that $\operatorname{dim}(R) \geq 2$ and that $H_{\mathfrak{m}}^{i}(R)$ is finitely generated $R$-module for all $i \neq \operatorname{dim}(R)$, then $R$ is Cohen-Macaulay.

The next result shows that $R \bowtie I$ is approximately Cohen-Macaulay if and only if $R$ is approximately Cohen-Macaulay provided some special conditions.

Proposition 3.2. Let $(R, \mathfrak{m})$ be a Noetherian local ring and let $I$ be a nonzero flat ideal of $R$. Assume that $R$ is not a Cohen-Macaulay ring such that $R$ is a homomorphic image of a Cohen-Macaulay local ring. Then $R \bowtie I$ is approximately Cohen-Macaulay if and only if $R$ is approximately CohenMacaulay.

Proof. Note that $\varphi: R \rightarrow R \bowtie I$ is a flat ring homomorphism. By [7, Proposition 5.1], we have $R \bowtie I / \mathfrak{m}_{0} \cong R / \mathfrak{m}$, where $\mathfrak{m}_{0}=\{(r, r+i) \mid r \in \mathfrak{m}, i \in I\}$ is the maximal ideal of $R \bowtie I$. So $R \bowtie I / \mathfrak{m}_{0}$ is a Cohen-Macaulay ring. Now the assertion follows from [13, Theorem 6$]$.

Before stating our main results of this section, we recall the definition of approximately Gorenstein ring due to Hochster in [10].

Definition 3.3. A Notherian local $\operatorname{ring}(R, \mathfrak{m})$ is called approximately Gorenstein, if for every integer $n>0$ there is an ideal $I \subseteq \mathfrak{m}^{n}$ such that $R / I$ is Gorenstein.

It is routine to see that every Gorenstein ring is approximately Gorenstein, and a zero dimensional ring is approximately Gorenstein if and only if it is Gorenstein. While approximately Gorenstein rings must have positive depth, they need not to be Cohen-Macaulay. In fact, every complete Noetherian domain is approximately Gorenstein [10, Theorem 1.6].

The next result shows that $R \bowtie I$ is approximately Gorenstein provided some special conditions.

Theorem 3.4. Let $(R, \mathfrak{m})$ be a Notherian local ring and let $I$ be a proper ideal of $R$. Then the following statements hold. 
(i) If $R$ is approximately Gorenstein, then $R \bowtie I$ is approximately Gorenstein.

(ii) If $R \bowtie I$ is Gorenstein and $R$ is generically Gorenstein, then $R$ is approximately Gorenstein.

Proof. (i) According to Proposition 2.1(3), $\left(R \bowtie I, \mathfrak{m}_{0}\right)$ is a Notherian local ring. Let $n>0$ be an integer. By assumption there exists an ideal $J \subseteq \mathfrak{m}^{n}$ such that $R / J$ is Gorenstein. By [7, Proposition 5.1], $J \bowtie I$ is an ideal of $R \bowtie I$ and

$$
\frac{R \bowtie I}{J \bowtie I} \cong \frac{R}{J}
$$

It is straightforward to see that $J \bowtie I \subseteq \mathfrak{m}^{n} \bowtie I=\mathfrak{m}_{0}^{n}$ and so $(R \bowtie I) /(J \bowtie I)$ is Gorenstein, therefore the assertion is proved.

(ii) By [1, Theorem 1.8], $R$ is Cohen-Macaulay and $I$ is a canonical ideal of $R$. The assertion follows from [10, Remarks (4.8b)].

Corollary 3.5. Let $R$ be a generically Gorenstein local ring and let $I$ be a proper ideal of $R$. Assume that $R$ is Cohen-Macaulay with canonical module. Then $R \bowtie I$ is approximately Gorenstein.

Proof. According to [10, Remarks (4.8b)], $R$ is approximately Gorenstein, so $R \bowtie I$ is approximately Gorenstein by Theorem 3.4(i).

Acknowledgment. We thank Siamak Yassemi for his detailed reading of this manuscript and many thoughtful suggestions.

\section{References}

[1] H. Ananthnarayan, L. Avramov, and W. Frank Moore, Connected sums of Gorenstein local rings, arXiv: 1005.1304v2 [math.AC] 10 Feb 2011.

[2] Y. Aoyama, Some basic results on canonical modules, J. Math. Kyoto Univ. 23 (1983), no. 1, 85-94.

[3] Y. Aoyama and S. Goto, On the endomorphism ring of the canonical module, J. Math. Kyoto Univ. 25 (1985), no. 1, 21-30.

[4] A. Bagheri, M. Salimi, E. Tavasoli, and S. Yassemi, A construction of quasi-Gorenstein rings, J. Algebra Appl, to appear.

[5] W. Bruns and J. Herzog, Cohen-Macaulay Rings, Cambridge University press, Cambridge, 1993.

[6] M. D'Anna, A construction of Gorenstein rings, J. Algebra 306 (2006), no. 2, 507-519.

[7] M. D'Anna, C. A. Finocchiaro, and M. Fontana, Amalgamated algebras along an ideal, Commutative algebra and its applications, 155-172, Walter de Gruyter, Berlin, 2009.

[8] M. D'Anna and M. Fontana, An amalgamated duplication of a ring along an ideal, J. Algebra Appl. 6 (2007), no. 3, 443-459.

[9] S. Goto, Approximately Cohen-Macaulay rings, J. Algebra 76 (1982), no. 1, 214-225.

[10] M. Hochster, Cyclic purity versus purity in excellent Noetherian ringss, Trans. Amer. Math. Soc. 231 (1977), no. 2, 463-488.

[11] H. Matsumura, Commutative Ring Theory, second ed., Studies in Advanced Mathemetics, vol.8, University Press, Cambridge, 1989.

[12] E. Platte and U. Storch, Invariante reguläre Differential-formen auf GorensteinAlgebren, Math. Z. 157 (1997), no. 1, 1-11. 
[13] M. R. Pournaki, M . Tousi, and S. Yassemi, Tensor products of approximately CohenMacaulay rings, Comm. Algebra 34 (2006), no. 8, 2857-2866.

[14] S. Yassemi, On flat and injective dimension, Ital. J. Pure Appl. Math. No. 6 (1999), $33-41$.

Elham Tavasoli

Department of Mathematics

SCIENCE AND RESEARCh BRANCH

ISLAMIC AZAD UNIVERSITY

TEHRAN, IRAN

E-mail address: elhamtavasoli@ipm.ir

MARYAM SALimi

Department of Mathematics

SCIENCE AND RESEARCH BRANCH

ISLAMIC AZAD UNIVERSITY

TEHRAN, IRAN

E-mail address: maryamsalimi@ipm.ir

ABOlFazl TeHranian

Department of Mathematics

SCIENCE AND Research BRANCH

ISLAMIC AZAD UNIVERSITY

TEHRAN, IRAN

E-mail address: tehranian@srbiau.ac.ir 